\title{
Single-fiber-laser-based wavelength tunable excitation for coherent Raman spectroscopy
}

\author{
Jue Su, ${ }^{1}$ Ruxin Xie, ${ }^{1}$ Carey K. Johnson, ${ }^{2}$ and Rongqing Hui ${ }^{1, *}$ \\ ${ }^{1}$ Department of Electrical Engineering and Computer Science, University of Kansas, Lawrence, Kansas 66045, USA \\ ${ }^{2}$ Department of Chemistry, University of Kansas, Lawrence, Kansas 66045, USA \\ ${ }^{*}$ Corresponding author: rhui@ku.edu
}

Received February 20, 2013; revised April 5, 2013; accepted April 25, 2013; posted May 1, 2013 (Doc. ID 185681); published May 27, 2013

\begin{abstract}
We demonstrate coherent Raman spectroscopy (CRS) using a tunable excitation source based on a single femtosecond fiber laser. The frequency difference between the pump and the Stokes pulses was generated by soliton self-frequency shifting in a nonlinear optical fiber. Spectra of $\mathrm{C}-\mathrm{H}$ stretches of cyclohexane were measured simultaneously by stimulated Raman gain (SRG) and coherent anti-Stokes Raman scattering (CARS) and compared. We demonstrate the use of spectral focusing through pulse chirping to improve CRS spectral resolution. We analyze the impact of pulse stretching on the reduction of power efficiency for CARS and SRG. Due to chromatic dispersion in the fiber-optic system, the differential pulse delay is a function of Stokes wavelength. This differential delay has to be accounted for when spectroscopy is performed in which the Stokes wavelength needs to be scanned. CARS and SRG signals were collected and displayed in two dimensions as a function of both the time delay between chirped pulses and the Stokes wavelength, and we demonstrate how to find the stimulated Raman spectrum from the two-dimensional plots. Strategies of system optimization consideration are discussed in terms of practical applications. (c) 2013 Optical Society of America

OCIS codes: (190.4370) Nonlinear optics, fibers; (300.6230) Spectroscopy, coherent anti-Stokes Raman

scattering; (300.6450) Spectroscopy, Raman; (320.1590) Chirping; (180.5655) Raman microscopy; (190.4223)

Nonlinear wave mixing.

http://dx.doi.org/10.1364/JOSAB.30.001671
\end{abstract}

\section{INTRODUCTION}

The spectral signature in Raman scattering can help reveal the molecular structures of materials, and therefore Raman microscopy is a powerful tool for label-free imaging for chemical and biomedical systems [1-3] . Compared to traditional spontaneous Raman scattering, coherent Raman scattering (CRS) can offer improved detection sensitivity and thus faster imaging. The nonlinear nature of CRS requires tight focusing of both the pump and the Stokes beams, which provides a viable solution to the need for three-dimensional optical sectioning inside thick samples [4]]. Different forms of CRS spectroscopy and microscopy have been demonstrated, including coherent anti-Stokes Raman scattering (CARS) [5, 6 ] and stimulated Raman scattering (SRS) $[4,7,8]$. While the intensity of the anti-Stokes signal measured by CARS includes contributions from both Raman resonances and the nonresonant background, SRS probes only the imaginary part of thirdorder nonlinear susceptibility, which is not affected by the nonresonant background.

Based on nonlinear mixing in the material, a CRS system requires two light sources as the pump and the Stokes beams with a frequency difference matching a vibrational frequency of the material to create efficient mixing. In addition, both the pump and the Stokes beams are usually short pulsed so that the average power is low to avoid damaging the sample, while the peak power can be high as required for nonlinear mixing. Precise temporal synchronization and the ability to continuously vary the frequency difference between the pump and the Stokes are two basic requirements for laser sources to probe the electronic vibrational modes of molecules in the sample [4-7]. A typical CRS setup uses either two feedback synchronized Ti:sapphire lasers or a Ti:sapphire laser and an optical parametric oscillator. The complexity of the laser system with the requirement of both temporal synchronization and wavelength tunability has so far limited the wide availability of CRS.

We have previously demonstrated CARS spectroscopy based on a single femtosecond fiber laser, which provided both the pump and the Stokes beams, and thus frequency synchronization between them was automatically maintained $[9,10]$. Fast wavelength tuning of Stokes pulses was accomplished by soliton self-frequency shifting (SSFS) in a photonic crystal fiber (PCF) $[11,12]$. Although CRS spectroscopy typically requires pulsewidths on the picosecond level, which best matches the spectral width of Raman lines, much shorter pulses on the femtosecond scale are needed for efficient frequency shifting by SSFS [11-14]. Theoretically the efficiency of frequency shifting through SSFS is inversely proportional to the fourth order of the temporal width of the pulse [13]. Unfortunately, the short temporal width and thus wide spectral linewidth of optical pulses would result in poor spectral resolution of CRS. Linear chirping, also known as spectral focusing, has been used to improve CRS spectral resolution [8, $\underline{15}-\underline{18}]$. But the increased temporal pulsewidth will decrease nonlinear mixing efficiency, leading to deterioration of the signal-to-noise ratio (SNR), so that the integration time will have to be increased in the measurement. Especially in a fiber-laser-based CRS system, in which signal optical power is 
a major limiting factor, the tradeoff between spectral resolution and power efficiency is an important issue in performance optimization.

Although CARS spectroscopy and imaging based on a single fiber laser have been demonstrated and investigated extensively $[10,19,20]$ and various fiber laser sources have been developed for this purpose [21-23], a systemic investigation and comparison between CARS and SRS based on such a fiber-laserbased system has not been reported and would help to better understand the capability of this system, as well as the design tradeoffs and performance optimization. In this paper, we demonstrate that both CARS and SRS can be performed using an excitation system based on a single fiber laser and a nonlinear wavelength scanner based on SSFS. The impact of pulse chirping and wavelength tuning on SRS and CARS are compared considering spectral resolution and SNR. Due to chromatic dispersion in the optical system, the differential pulse delay is a function of the Stokes wavelength. This differential delay must be taken into account when spectroscopy is performed in which the Stokes wavelength is scanned. We introduce two-dimensional plots of the CARS and stimulated Raman gain (SRG) signals in which the signal is displayed as a function of time delay and Stokes wavelength, and we show how to extract the spectrum as a cut in the two-dimensional plot. Strategies of system optimization consideration are discussed in terms of practical applications.

\section{THEORETICAL BACKGROUND}

Theoretically both CARS and SRS originate from the thirdorder polarization due to the nonlinear interaction between the excitation fields and the third-order nonlinear susceptibility of the material. In the frequency domain, the third-order nonlinear susceptibility of a material can be expressed as [1]

$$
\chi^{(3)}(\omega)=\sum_{m} \frac{A_{m}}{\Omega_{m}-\omega-i \Gamma_{m} / 2}+\chi_{\mathrm{nr}}^{(3)},
$$

where $A_{m}$ is proportional to the spontaneous Raman cross section, $\Gamma_{m}$ is the full width at half-maximum (FWHM), $\Omega_{m}$ is the Raman vibrational frequency of the $m$ th vibrational mode, $\omega$ is the frequency, and $\chi_{\mathrm{nr}}^{(3)}$ represents the nonresonant background, which is independent of $\omega$.

In the CARS process, mixing between the pump and the complex conjugate of the Stokes pulse provides a stimulus for the nonlinear susceptibility to generate the response,

$$
\tilde{R}_{s p}(\omega)=\operatorname{FT}\left[\tilde{E}_{p}(t) \cdot \tilde{E}_{s}^{*}(t)\right] \cdot L(\omega),
$$

where $\tilde{E}_{p}(t)$ and $\tilde{E}_{s}(t)$ are the time-domain electric fields of the chirped pump and Stokes pulses, and

$$
L(\omega)=\sum_{m} \frac{B_{m}}{\Omega_{m}-\omega-j \Gamma_{m} / 2}
$$

with $B_{m}$ a proportionality constant. FT is the Fourier transform, and $*$ represents the complex conjugate. With appropriate frequency separation between the pump and the Stokes, the spectral density of $\tilde{R}_{s p}(\omega)$ is nonzero only in the vicinity of molecular vibrational frequency $\Omega_{m}$. The pump also acts as a probe, to generate an anti-Stokes wave given in the frequency domain by

$$
\tilde{E}_{\mathrm{AS}}(\omega)=\operatorname{FT}\left[\tilde{R}_{s p}(t) \cdot \tilde{E}_{p}(t)\right]
$$

where $\tilde{R}_{s p}(t)=\operatorname{IFT}\left[\tilde{R}_{s p}(\omega)\right]$ is the stimulated resonance in the time-domain, IFT is the inverse Fourier transform, and $\tilde{E}_{p}(t)$ is the chirped probe (pump) pulse. The optical power spectral density of the anti-Stokes wave can be detected directly by a spectrometer, and the photocurrent is

$$
I_{C}(\omega)=\eta(\omega)\left|\tilde{E}_{\mathrm{AS}}(\omega)\right|^{2} \propto\left|\chi_{1111}^{(3)}\right|^{2} \cdot\left|A_{p}\right|^{4}\left|A_{s}\right|^{2}
$$

where $\eta(\omega)$ is the frequency-dependent responsivity of the photodetector and $A_{p}$ and $A_{s}$ are the amplitudes of the pump and Stokes pulses.

In the SRS process, on the other hand, the pump and the Stokes pulses interact through the imaginary part of the third-order nonlinear susceptibility of the material, with the response

$$
\tilde{R}_{s p}^{\prime}(\omega)=\operatorname{FT}\left[\tilde{E}_{p}(t) \cdot \tilde{E}_{s}^{*}(t)\right] \cdot j L(\omega)
$$

This nonlinear response mixes with the complex conjugate of the probe, producing an electrical field at the Stokes frequency $\omega_{s}=\omega_{p}-\Omega_{m}$, which is equivalent to a parametric gain for the Stokes and is linearly proportional to the power of the pump and the field of the Stokes as

$$
\tilde{E}_{\mathrm{SRG}}(\omega)=\mathrm{FT}\left[\tilde{R}_{s p}^{\prime}(t) \cdot \tilde{E}_{p}^{*}(t)\right]
$$

Since $\tilde{E}_{\mathrm{SRS}}(\omega)$ has the same frequency as the Stokes field, it can be measured through a coherent homodyne detection process after mixing with the relatively strong Stokes field at the photodetector. The photocurrent generated in this coherent detection process is

$$
I(t)=\eta_{\omega}\left|\tilde{E}_{s}(t)+\tilde{E}_{\mathrm{SRS}}(t)\right|^{2},
$$

where $\eta_{\omega}$ is the photodetector responsivity at the Stokes wavelength and $\tilde{E}_{\mathrm{SRS}}(t)=\operatorname{IFT}\left[\tilde{E}_{\mathrm{SRS}}(\omega)\right]$ is the time domain response of SRS induced parametric gain. Since the interaction length inside the sample is usually very short, of the order of $<1 \mu \mathrm{m}$, the parametric gain introduced through the SRS is very weak, of the order of $10^{-5}$, and the detection of this small perturbation is usually challenging. A commonly used technique for SRG microscopy is to use an intensity modulated pump so that the intensity of $\tilde{E}_{\mathrm{SRS}}(t)$ is modulated at the same frequency. Assuming that the amplitude and phase of the Stokes field are constant, and neglecting the dc components in the photocurrent, the time-varying photocurrent is

$$
i_{\mathrm{SRS}}(t)=2 \eta_{\omega} \tilde{E}_{s} \operatorname{Re}\left[\tilde{E}_{\mathrm{SRS}}(t)\right] \propto \operatorname{Im}\left[\chi_{1111}^{(3)}\right] \cdot\left|A_{p}\right|^{2}\left|A_{s}\right|^{2}
$$

This can be detected by using a lock-in amplifier synchronized with the modulating waveform of the pump.

Given the pulse shapes $A_{p}(t)$ and $A_{s}(t)$, and the central frequencies $\omega_{p}$ and $\omega_{s}$ for the pump and Stokes fields, numerical simulations can be performed based on Eqs. (1)-(9). The impact of various system parameters can be considered, including pulsewidth, pulse shape and chirp, as well as the differential pulse delay caused by chromatic dispersion. This provides a useful tool for system performance comparison and optimization. 
Apart from numerical simulations, analytical formulas can also be derived to elaborate general rules. Assuming both the pump and the Stokes pulses are chirped Gaussian pulses, their optical fields can be expressed as $[\underline{16}, \underline{17}]$

$$
\tilde{E}_{p}(t)=A_{p 0}\left[\frac{\Delta_{p}^{2} \pi}{(\ln 2)\left(1+C_{p}^{2}\right)}\right]^{1 / 4} \exp \left[-\frac{\left(1+j C_{p}\right) t^{2} \Delta_{p}^{2} \pi^{2}}{2 \ln 2} \frac{1+C_{p}^{2}}{\ln }\right] \exp \left(j \omega_{p} t\right),
$$

$\tilde{E}_{s}(t)=A_{s 0}\left[\frac{\Delta_{s}^{2} \pi}{(\ln 2)\left(1+C_{s}^{2}\right)}\right]^{1 / 4} \exp \left[-\frac{\left(1+j C_{s}\right)}{2 \ln 2} \frac{t^{2} \Delta_{s}^{2} \pi^{2}}{1+C_{s}^{2}}\right] \exp \left(j \omega_{s} t\right)$,

where $A_{p 0}$ and $A_{s 0}$ are field amplitudes, and $\Delta_{p}$ and $\Delta_{s}$ are the FWHM spectral widths of the pump and the Stokes. $C_{p}$ and $C_{s}$ are chirp parameters defined as

$$
\begin{aligned}
& C_{p}=\sqrt{\left(T_{\mathrm{pc}}^{2} / T_{p 0}^{2}\right)-1}, \\
& C_{s}=\sqrt{\left(T_{\mathrm{sc}}^{2} / T_{s 0}^{2}\right)-1}
\end{aligned}
$$

where $T_{p 0}$ and $T_{s 0}$ are the FWHM temporal widths of transform limited Gaussian pulses, related to the spectral width by $T_{p 0, s 0}=(2 \ln 2) /\left(\pi \Delta_{p, s}\right) . T_{\mathrm{pc}}$ and $T_{\mathrm{sc}}$ are FWHM widths for the chirped pump and Stokes pulses, respectively.

The spectral resolution of CRS primarily depends on the mixing between the pump and the Stokes pulses, and their mixing product is also linearly chirped,

$$
\begin{aligned}
\tilde{E}_{p}(t) \tilde{E}_{s}^{*}(t)= & E_{m 0} \exp \left\{\frac { - t ^ { 2 } \pi ^ { 2 } } { 2 \operatorname { l n } 2 } \left[\left(\frac{\Delta_{p}^{2}}{1+C_{p}^{2}}+\frac{\Delta_{s}^{2}}{1+C_{s}^{2}}\right)\right.\right. \\
& \left.\left.+j\left(\frac{C_{p} \Delta_{p}^{2}}{1+C_{p}^{2}}-\frac{C_{s} \Delta_{s}^{2}}{1+C_{s}^{2}}\right)\right]\right\} \exp \left[j\left(\omega_{p}-\omega_{s}\right) t\right] \\
= & E_{p 0} E_{s 0} \exp \left[-\frac{\left(1+j C_{\text {eff }}\right.}{2 \ln 2} \frac{t^{2} \pi^{2} \Delta_{\text {eff }}^{2}}{1+C_{\text {eff }}^{2}}\right] \\
& \times \exp \left[j\left(\omega_{p}-\omega_{s}\right) t\right],
\end{aligned}
$$

where

$$
C_{\text {eff }}=\frac{C_{p} \Delta_{p}^{2}\left(1+C_{s}^{2}\right)-C_{s} \Delta_{s}^{2}\left(1+C_{p}^{2}\right)}{\Delta_{p}^{2}\left(1+C_{s}^{2}\right)+\Delta_{s}^{2}\left(1+C_{p}^{2}\right)}
$$

is the effective chirp parameter,

$$
E_{m 0}=A_{p 0} A_{s 0} \sqrt{\frac{\pi \Delta_{p} \Delta_{s}}{\ln 2}}\left(\frac{1}{\left(1+C_{p}^{2}\right)\left(1+C_{s}^{2}\right)}\right)^{1 / 4}
$$

is the magnitude, and

$$
\Delta_{\text {eff }}=\sqrt{\left(\frac{\Delta_{p}^{2}}{1+C_{p}^{2}}+\frac{\Delta_{s}^{2}}{1+C_{s}^{2}}\right)\left(1+C_{\mathrm{eff}}^{2}\right)}
$$

is the effective linewidth of the mixing product, which provides an estimation of the spectral resolution for CRS when both the pump and the Stokes pulses are linearly chirped. Figure 1 is a contour plot that shows $\Delta_{\text {eff }}$ as a function of the stretched temporal widths of the pump and Stokes pulses. Assuming that the unchirped pulses have pulsewidths $T_{p 0}=T_{s 0}=100 \mathrm{fs}$, the corresponding CRS spectral resolution is approximately $208 \mathrm{~cm}^{-1}$. To achieve a $20 \mathrm{~cm}^{-1}$ spectral resolution, the pump and the Stokes pulses have to be chirped to at least $1040 \mathrm{fs}$, corresponding to a chirp parameter of approximately $C_{p}=C_{s}=10.35$. It is also important to note from Fig. 1 that identical chirping for the pump and the Stokes is required in order to achieve the best spectral resolution. This is especially true for highly chirped pulses, where even a small mismatch of chirping rate will cause significant degradation of CRS spectral resolution.

Note that although spectral focusing through an identical chirp applied on both the pump and the Stokes pulses improves the spectral resolution, for fixed average optical power, temporally stretched pulses will inevitably reduce the nonlinear mixing efficiency and result in reduced CRS signals. It is generally accepted that the strength of the anti-Stokes signal generated by the CARS process is proportional to $\left|E_{p}\right|^{4}\left|E_{s}\right|^{2}$, while the coherently detected SRS signal at the Stokes frequency is proportional to $\left|E_{p}\right|^{2}\left|E_{s}\right|^{2}$. If the receivers are ideally shot-noise limited, the SNRs will be proportional to $\left|E_{p}\right|^{2}\left|E_{s}\right|$ and $\left|E_{p}\right|\left|E_{s}\right|$ for CARS and SRS, respectively [24]. As CRS involves spectral convolution between the pump and the Stokes pulses and the material nonlinear susceptibility, it is important to understand the impact of pulse chirping on these scaling rules, which are obviously different from using transform-limited pump and Stokes pulses. Note that since CARS measures $\left|\chi_{1111}^{(3)}\right|^{2}$, the nonresonant background may have a significant impact on the CARS spectral shape. However, this impact is deterministic, depending on the nature of the material under test. For generality, the impact of the nonresonant background is not included as part of the random noise in our SNR consideration. In fact, techniques exist to extract the resonant imaginary component of $\chi_{1111}^{(3)}$ from CARS measurements [25,26].

Neglecting the frequency response of the material nonlinear susceptibility, the anti-Stokes field in the four-wave mixing process is proportional to

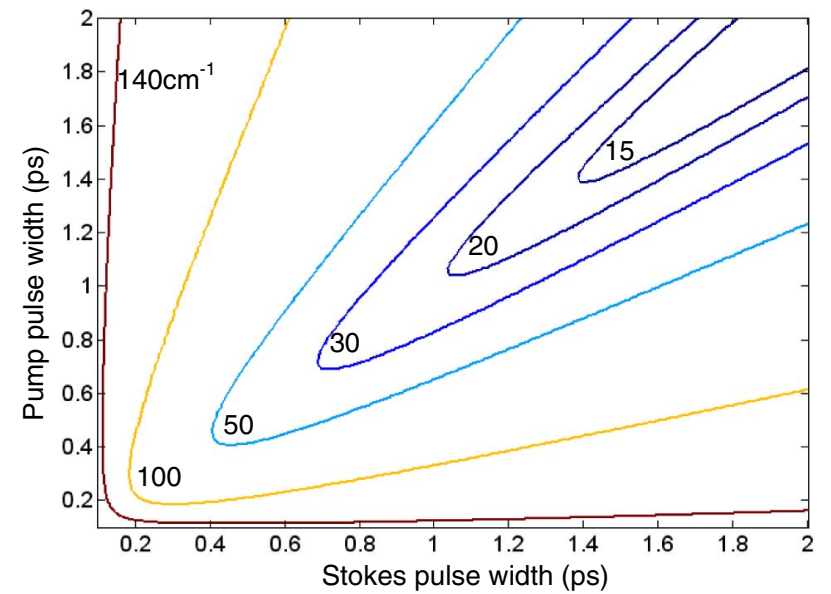

Fig. 1. Contour plot of calculated SRS spectral resolution (indicated by the value near each curve) as a function of chirped pump and Stokes pulse temporal widths. The unchirped pump and Stokes pulses are both transform-limited with 0.1 ps width. 


$$
\begin{aligned}
\tilde{E}_{p}^{2}(t) \tilde{E}_{s}^{*}(t)= & E_{0 C} \exp \left[-\frac{t^{2} \pi^{2} \Delta_{\text {eff }}^{2}}{2 \ln 2\left(1+C_{\text {eff }}^{2}\right)}\left(1+j C_{\text {eff }}\right)\right] \\
& \times \exp \left[j\left(2 \omega_{p}-\omega_{s}\right) t\right]
\end{aligned}
$$

where

$$
E_{0 C}=A_{p 0}^{2} A_{s 0}\left[\frac{\Delta_{p}^{2} \pi}{(\ln 2)\left(1+C_{p}^{2}\right)}\right]^{1 / 2}\left[\frac{\Delta_{s}^{2} \pi}{(\ln 2)\left(1+C_{s}^{2}\right)}\right]^{1 / 4}
$$

is the field peak amplitude,

$$
C_{\text {eff }}=\frac{2 C_{p} \Delta_{p}^{2}\left(1+C_{s}^{2}\right)-C_{s} \Delta_{s}^{2}\left(1+C_{p}^{2}\right)}{2 \Delta_{p}^{2}\left(1+C_{s}^{2}\right)+\Delta_{s}^{2}\left(1+C_{p}^{2}\right)}
$$

is the effective chirp parameter, and

$$
\Delta_{\text {eff }}=\sqrt{\left(\frac{2 \Delta_{p}^{2}}{1+C_{p}^{2}}+\frac{\Delta_{s}^{2}}{1+C_{s}^{2}}\right)\left(1+C_{\mathrm{eff}}^{2}\right)}
$$

is the effective spectral linewidth. Integrating the power spectral density function of the anti-Stokes wave, the total optical power of the anti-Stokes is then

$$
P_{\mathrm{CARS}}=E_{0 c}^{2} \sqrt{4 \pi \ln 2} \sqrt{\frac{1+C_{\mathrm{eff}}^{2}}{\Delta_{\mathrm{eff}}^{2}}}
$$

For SRS, the mixing signal through coherent detection is proportional to $\left|\tilde{E}_{p}(t)\right|^{2}\left|\tilde{E}_{s}(t)\right|^{2}$. Integrating over the pulse duration yields the SRS signal amplitude,

$$
A_{\mathrm{SRS}}=A_{p 0}^{2} A_{s 0}^{2} \sqrt{\frac{\pi}{\ln 2}} \sqrt{\frac{\Delta_{p}^{2} \Delta_{s}^{2}}{\Delta_{p}^{2}\left(1+C_{s}^{2}\right)+\Delta_{s}^{2}\left(1+C_{p}^{2}\right)}} .
$$

The contour plots shown in Fig. 2 indicate signal reductions for CARS and SRS as a function of chirped pulsewidths obtained with Eqs. (22) and (23). The original temporal width of the pump and the Stokes are both $100 \mathrm{fs}$. In the ideal case of identical chirp for the pump and the Stokes pulses, $\Delta_{p}=$ $\Delta_{s}=\Delta$ and $C_{p}=C_{s}=C$ as indicated on the dashed straight lines in the figure; the CARS signal power is proportional to
$\left(T_{0} / T_{1}\right)^{2}$, while the SRS signal amplitude is linearly proportional to $T_{0} / T_{1}$. For example, when both the pump and the Stokes pulses are chirped from 0.1 to $1 \mathrm{ps}$, the anti-Stokes power is reduced by $20 \mathrm{~dB}$, while the SRS signal is reduced by only $10 \mathrm{~dB}$.

It is important to note that in the calculation to obtain Eqs. (22) and (23) the spectral shape of the Raman line was not considered, or, equivalently, its linewidth was assumed to be much wider than the spectral width of the excitation optical pulses. In practical applications, especially those using femtosecond optical pulses, the spectral width of the pulses can be much wider than the Raman linewidth, which is typically less than $50 \mathrm{~cm}^{-1}$, so that a significant part of the excitation optical spectral width may not participate in the resonant nonlinear mixing process, reducing the strength of the CRS signal. As a result, the impact of pulse chirping on CRS signal amplitude will be modified from those predicted by Eqs. (22) and (23), depending on the actual linewidth and line shape of the resonant Raman response.

Since the third-order nonlinear susceptibility typically exhibits a Lorentzian spectral line shape as indicated by Eq. (1), it is not convenient to derive simple analytical expressions by convolving with Gaussian pulses. By numerical integration, it is straightforward to find the impact of pulse chirping on the CARS and SRS signal amplitudes and SNR. Figure 3 shows the relative signal reduction as a function of the chirped pump and Stokes pulsewidths, where the original temporal width of the pump and the Stokes were both $100 \mathrm{fs}$. In this calculation, we assumed that there is only one Raman line, and the nonresonant background was neglected. The contour plots in Figs. 3(a) and 3(b) show the relative signal reduction of CARS and SRS, respectively, when the spectral width of the Raman line is $9 \mathrm{~cm}^{-1}$. The results shown in Fig. 3 are significantly different compared to those in Fig. 2, and the signal reduction caused by pulse chirping becomes much less. The major reason is that with a narrow Raman line spectral width, a large portion of the spectrum in the original $100 \mathrm{fs}$ excitation pulses does not contribute to the nonlinear mixing process within the narrow Raman line, and therefore increased chirping does not significantly reduce the mixing efficiency. Another important observation from Figs. 3(a) and 3(b) is that the highest mixing efficiency (the least amplitude reduction) happens when the pump and the
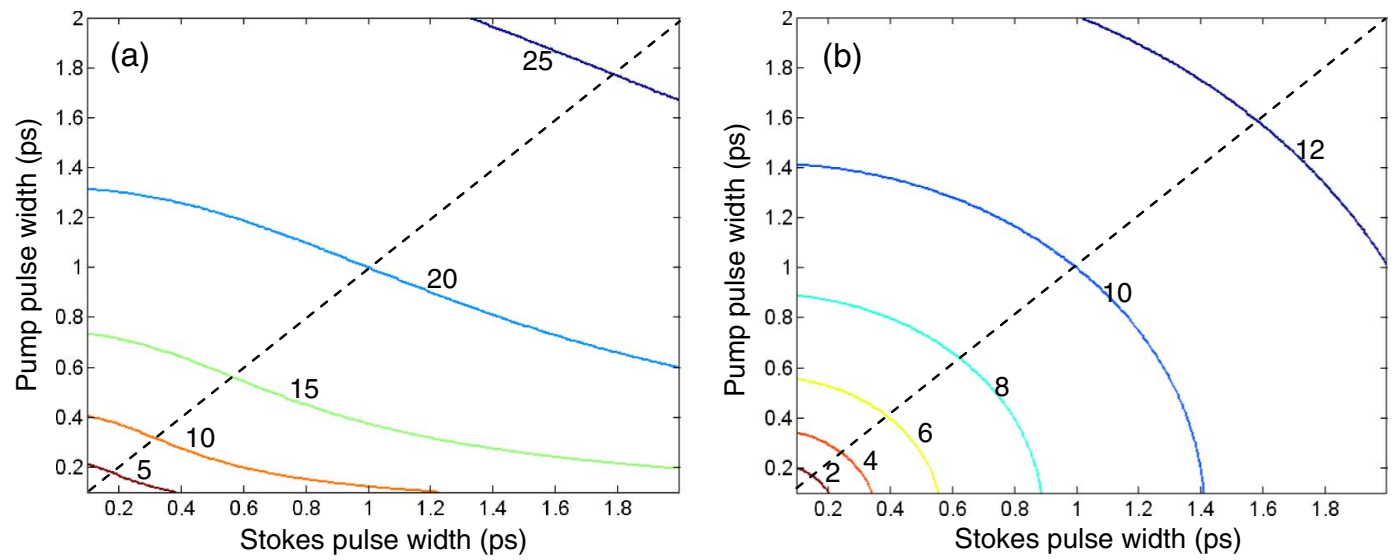

Fig. 2. Contour plots of calculated CRS signal reduction in decibels (marked by the value near each curve) based on Eq. (22) for CARS (a) and Eq. (23) for SRG (b), as a function of the chirped pump and Stokes pulse temporal widths. The unchirped pump and Stokes pulses were both transform-limited with $0.1 \mathrm{ps}$ width. Dashed straight lines indicate equally chirped pump and Stokes pulses. 

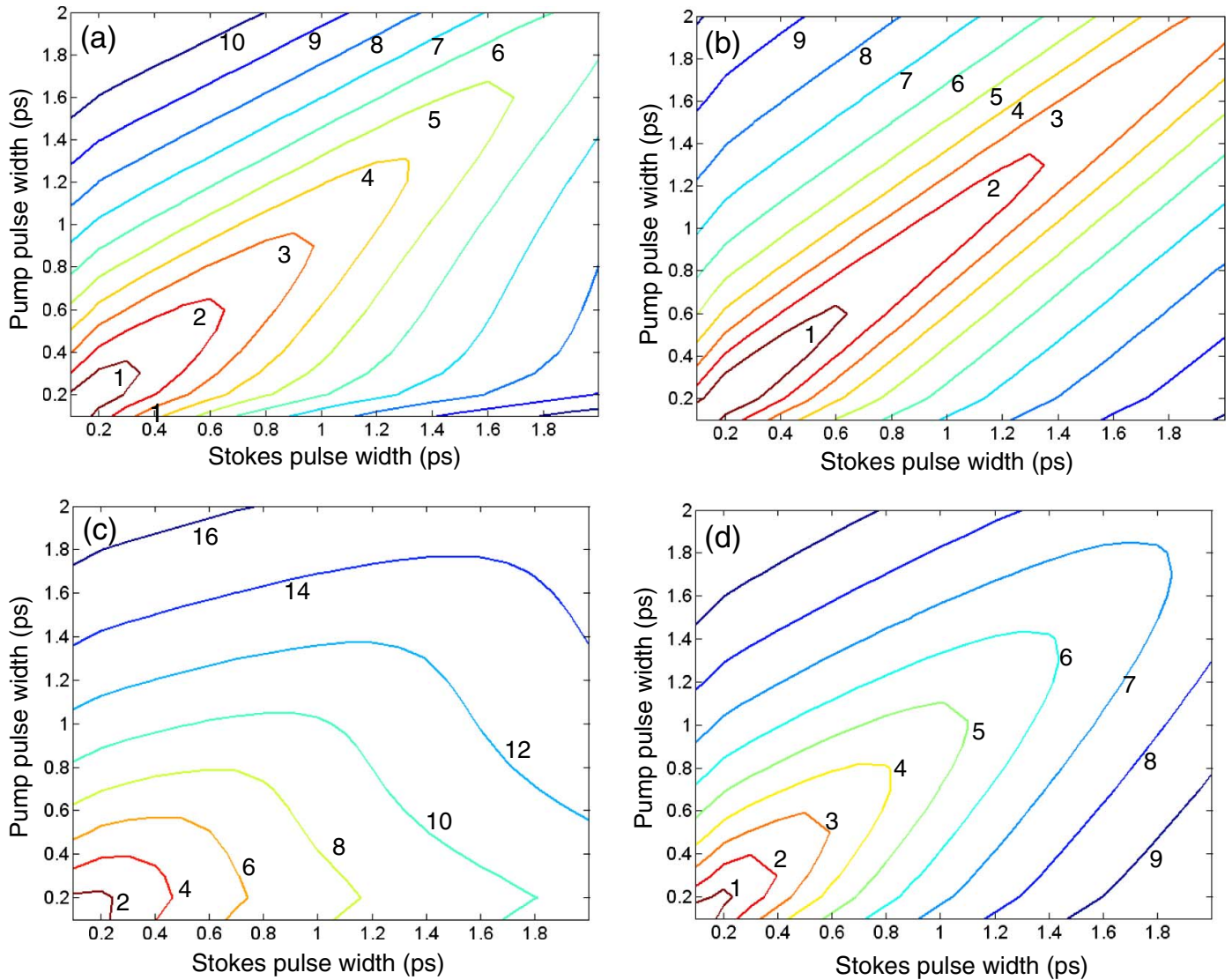

Fig. 3. Contour plot of (a) and (c) CARS, and (b) and (d) SRG signal reduction in decibels (marked by the value near each curve) as a function of the chirped pump and Stokes pulse temporal widths. The unchirped pump and Stokes pulses are both transform-limited with 0.1 ps width. (a) and (b) are for Raman resonance linewidth of $9 \mathrm{~cm}^{-1}$, and (c) and (d) are for a $45 \mathrm{~cm}^{-1}$ linewidth.

Stokes are identically chirped so that their slopes in the timefrequency diagram are identical. When the spectral width of the Raman line is wider, the mixing efficiency is higher with the original 100 fs transform-limited pulses without chirping, and therefore the relative CRS signal amplitude degradation appears more severe with chirping as shown in Figs. 3(c) and $3(\mathrm{~d})$, where the spectral width of the Raman line is increased to $45 \mathrm{~cm}^{-1}$. When the Raman linewidth is increased to $>500 \mathrm{~cm}^{-1}$, the calculated contour plots using numerical integration become nearly identical to those using Eqs. (22) and (23) shown in Fig. 2.

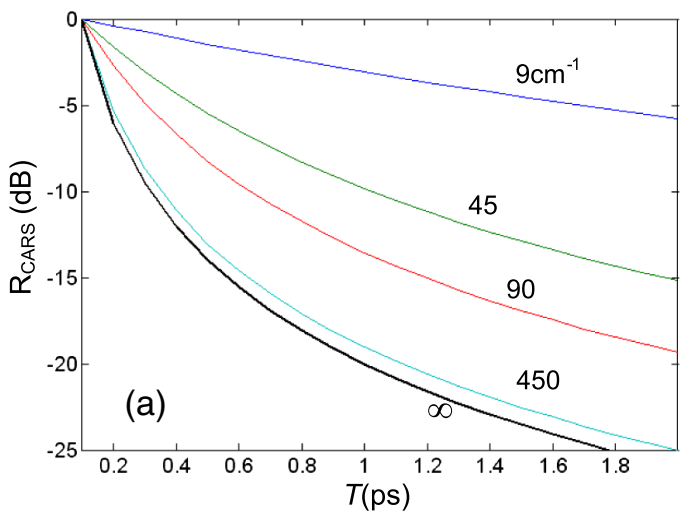

In the ideal case when the pump and the Stokes are chirped identically, both the spectral resolution and the mixing efficiency are optimized as indicated by Figs. 1 and 3. Figures 4(a) and 4(b) show the relative amplitude reduction for the $\overline{\mathrm{CARS}}$ and the SRS signals, respectively, when the pump and the Stokes are identically chirped for different spectral width of the Raman lines. It is evident that as the spectral linewidth of the nonlinear susceptibility is reduced, the signal amplitudes for both CARS and SRS become less affected by pulse chirping, especially for SRS, where only a $2.5 \mathrm{~dB}$ signal amplitude reduction is introduced when the pump and Stokes

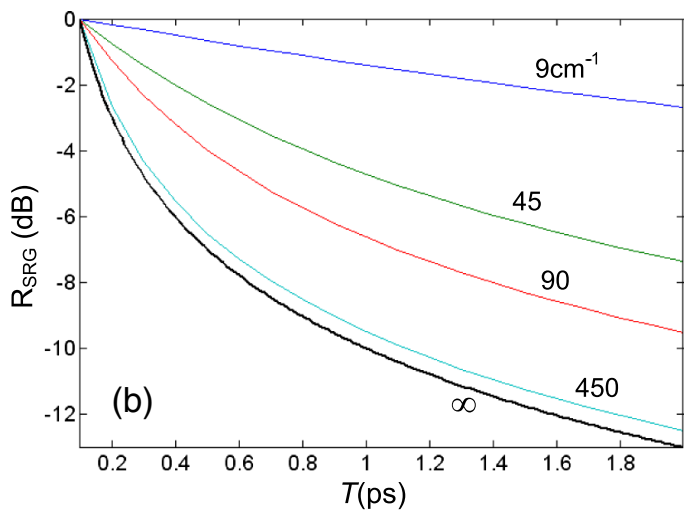

Fig. 4. Calculated relative signal reduction as the function of chirped pulsewidth (identical for the pump and the Stokes) for different spectral widths of the Raman spectral line $\left(9,45,90\right.$, and $450 \mathrm{~cm}^{-1}$ ), (a) for CARS and (b) for SRS. 
pulses are chirped from 0.1 to 2 ps for a Raman linewidth of $9 \mathrm{~cm}^{-1}$. For CARS, the corresponding signal reduction is approximately $6 \mathrm{~dB}$. Since nonresonant background in CARS has very broad spectral width, Fig. 4(a) suggests that pulse chirping reduces nonresonant background much more than it does the Raman lines.

Another possible way to increase CRS spectral resolution is to apply narrowband optical filters on both the pump and the Stokes beams so that their pulsewidths can be stretched in the time domain [27]. However, this would significantly reduce the optical power for both the pump and the Stokes. For the same stretched pulsewidth, the reduction in the CARS and SRS signal amplitude can be several orders of magnitude more than obtained by pulse chirping. In fact, without considering the linewidth of the nonlinear susceptibility, the signal amplitude reduction is proportional to $\left(T_{0} / T_{1}\right)^{5}$ for CARS, and $\left(T_{0} / T_{1}\right)^{3}$ for SRS when spectral limiting optical filters are used. From a practical application point of view, for a fiber-laser-based CRS excitation system in which optical power is a primary concern, pulse chirping is obviously a better choice in comparison to optical filtering.

Note that in the analysis we have considered only a single Raman line in the material and have neglected the nonresonant background. In general, since SRS measures the imaginary part of the third-order nonlinear susceptibility of the material, unequal chirping rates between the pump and the Stokes only results in reduced spectral resolution. On the other hand, CARS measures the absolute value of the thirdorder nonlinear susceptibility of the material when the pump and the Stokes pulses are chirped by the same rate. If they are chirped differently, not only is the spectral resolution reduced, but the measured spectral line shape may also be distorted, especially when the spectral resolution is not high enough, as discussed further below.

\section{EXPERIMENTS}

We used the $\mathrm{C}-\mathrm{H}$ band of liquid cyclohexane as the sample for the measurements because of its well-known Raman spectrum. Figure $\underline{5}$ shows the spontaneous Raman spectrum of cyclohexane measured with a confocal Raman spectrometer (Horiba Jobin Yvon LabRam ARAMIS). The solid curve in Fig. $\underline{5}$ is a numerical fitting of the measured spectrum

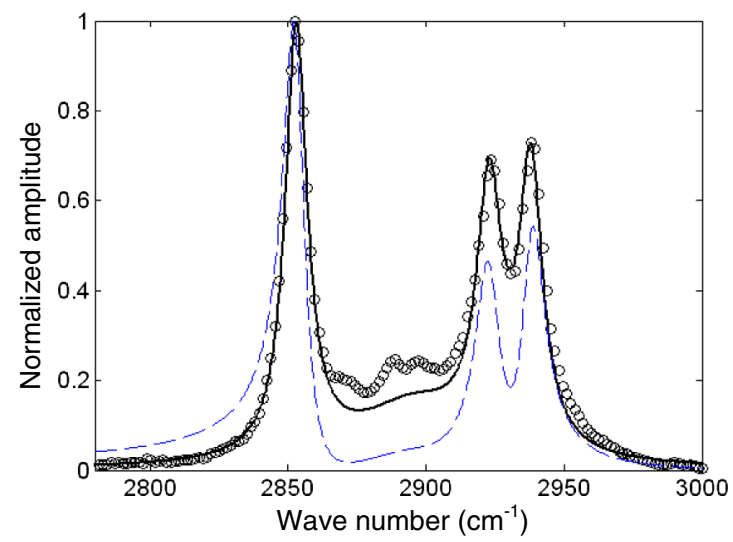

Fig. 5. Measured (open circles) spontaneous Raman spectrum and numerical fitting (solid curve) with the imaginary part of Eq. (1). The dashed curve represents $\left|\chi_{1111}^{(3)}\right|^{2}$ deduced from the spontaneous Raman spectrum and with nonresonant background added. with the imaginary part of Eq. (1) by adjustment of the Raman cross section and the damping rate of each Raman line. The three major Raman lines are at 2853, 2923, and $2938 \mathrm{~cm}^{-1}$, with FWHM linewidths of $9.6,11.2$, and $10.1 \mathrm{~cm}^{-1}$, respectively. These parameters were used in the calculation and compared with CRS measurements. We also assumed $2.5 \%$ nonresonant background; although it has no impact on the SRS spectrum, it affects the line shape of CARS spectroscopy, which measures $\left|\chi_{1111}^{(3)}\right|^{2}$ shown as the dashed curve in Fig. 5 .

To investigate the improvement of CRS spectral resolution through pulse chirping and demonstrate CARS and SRS spectroscopy using a low power budget fiber laser, we have conducted an experiment using the setup shown in Fig. $\underline{6}$. A fiber laser (IMRA Femtolite-100) was used as the light source, which generated $120 \mathrm{fs}$ pulses at $802 \mathrm{~nm}$ with a $9.5 \mathrm{~nm}$ spectral linewidth. The average optical power emitted from the laser was approximately $100 \mathrm{~mW}$ with a pulse repetition rate of $75 \mathrm{MHz}$, corresponding to a pulse energy of about $1.3 \mathrm{~nJ}$. This laser output was split into the pump and the Stokes paths. The pump passed through a motorized delay stage (Opto-Sigma) and a $5 \mathrm{~cm}$-long glass rod (Schott SF6) for pulse chirping. A Pockels cell (Conoptics) followed by a polarizer was used as an intensity modulator so that the pump pulse could be rapidly switched on and off. The Stokes path had a voltagecontrolled variable optical attenuator (VOA), which controlled the optical power that entered a $2 \mathrm{~m}$ long PCF (Crystal Fiber NL-PM-750). By changing the voltage applied on the VOA, the wavelength of the Stokes could be varied from 850 to $1200 \mathrm{~nm}$ through SSFS in the PCF. Figure 7(a) shows an example of measured spectra when the Stokes was shifted to different wavelengths.

The pulsewidth of the frequency-shifted fundamental soliton was approximately 90 fs with a spectral width of $17.25 \mathrm{~nm}$ at a Stokes wavelength of $1040 \mathrm{~nm}$. A long-pass filter after the PCF blocked the residual power at $802 \mathrm{~nm}$ as well as higherorder solitons generated below $850 \mathrm{~nm}$. In this case the average power launched at the PCF input was about $20 \mathrm{~mW}$ (without considering fiber coupling efficiency), and the average power of the fundamental soliton was approximately $3.5 \mathrm{~mW}$. Another $5 \mathrm{~cm}$ SF6 glass rod was used in the Stokes path with its end surfaces partially coated with gold, so that the Stokes wave could fold back multiple times through the rod. The pump and the Stokes pulses were recombined with a dichroic beam combining filter and focused at the sample by an objective lens (Nikon 40x). Average optical powers at the focal point were approximately $13 \mathrm{~mW}$ for the pump and $0.7 \mathrm{~mW}$ for the Stokes. The major contribution to power loss was from the objectives, which were not optimized for nearinfrared wavelengths. For SRS measurements, a long-pass filter eliminated the pump so that only the Stokes beam was detected by a large area InGaAs photodetector (TIA5251) with $125 \mathrm{MHz}$ bandwidth. A lock-in amplifier (Stanford Research Systems SR-850) triggered by the same waveform that was used to drive the Pockels cell detected the SRG component in the Stokes. For CARS measurements, a bandpass filter rejected both the pump and the Stokes wavelengths, and the intensity of the anti-Stokes was captured by a spectrometer (Ocean Optics, Maya 2000 pro). A laptop computer was used to control the Stokes wavelength through the VOA and the relative pulse delay between the pump and the Stokes pulses through the motorized delay stage, to synchronize the 


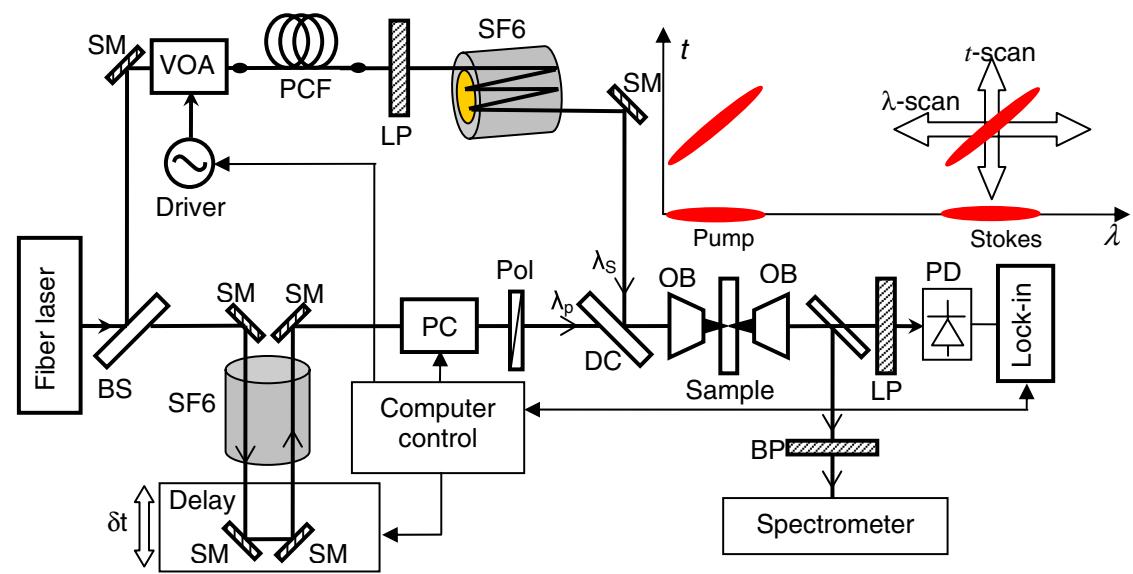

Fig. 6. Experimental configuration with controllable Stokes wavelength $\left(\lambda_{S}\right)$ and pump relative delay $(\delta t)$. BS, beam splitter; SM, silver mirror; PCF, photonic crystal fiber; LP, long-pass filter; BP, bandpass filter; SF6, SF-6 glass rod; DC, dichroic beam combiner; OB, objective lenses; PC, Pockels cell; Pol, polarizer; PD, photodiode. Inset, illustration of time-wavelength diagram of pump and Stokes waves.

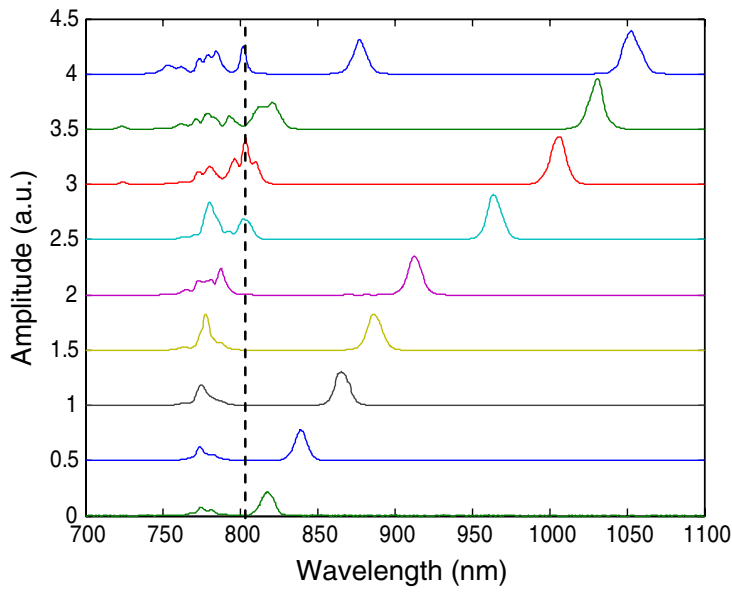

(a)

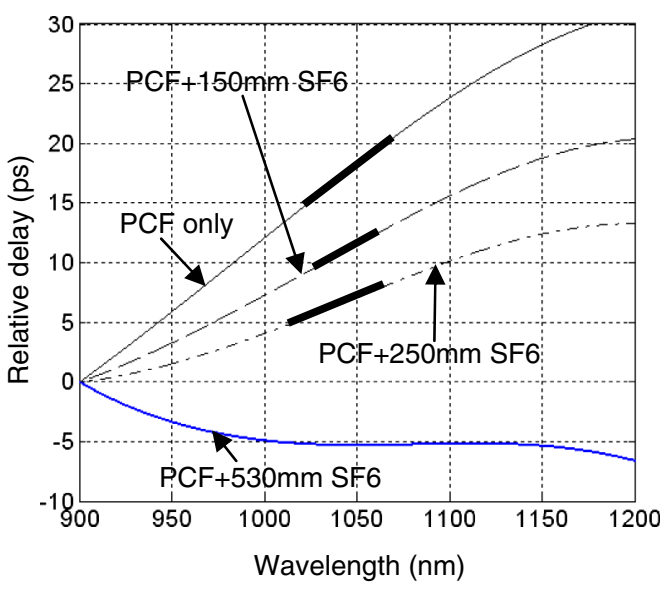

(b)

Fig. 7. (a) Measured Stokes spectra with wavelength shifted by SSFS. The curves are shifted by 0.5 between one and the others for better display. The vertical dashed line indicates the original laser wavelength before shifting. (b) Calculated relative delay of the Stokes pulse as the function of wavelength (relative to the delay at $900 \mathrm{~nm}$ ). Short sections marked with bold-lines indicate slopes used in the measurements shown in Figs. 9-11.

driving waveform for the Pockels cell and the lock-in amplifier, and to perform data acquisition from the lock-in amplifier and the spectrometer. After passing through the Pockels cell, the pump pulses were stretched to approximately $175 \mathrm{fs}$. Because of the chromatic dispersion in the PCF, the pulse propagation delay is also a function of wavelength. Figure $7(\mathrm{~b})$ shows the calculated pulse delays relative to their values at a $900 \mathrm{~nm}$ wavelength. Since the PCF has anomalous dispersion in the vicinity of the $1 \mu \mathrm{m}$ wavelength while the SF6 glass has normal dispersion in that region, adding SF6 glass for pulse chirping reduces the overall differential group delay for the Stokes pulses during wavelength tuning. Figure 7 also shows that the differential group delay can be minimized in a wide wavelength window with optimum design. Specifically, using a $2 \mathrm{~m}$ PCF and $530 \mathrm{~mm}$ of SF6 glass, the variation of the Stokes wave group delay can be less than $0.5 \mathrm{ps}$ in the window from 1000 to $1150 \mathrm{~nm}$, corresponding to a frequency window of $1300 \mathrm{~cm}^{-1}$.

In this setup, wavelength tuning of the Stokes pulses was accomplished by SSFS in a nonlinear fiber. Although the pulsewidth of the fundamental soliton does not change significantly when the wavelength is varied, chromatic dispersion in the fiber introduces pulse propagation delay as a function of wavelength. This differential delay has to be compensated by the tunable delay stage in the pump path during the measurement. In our experimental setup without pulse chirping, the differential pulse delay of about $0.121 \mathrm{ps} / \mathrm{nm}$ was created primarily by the PCF. We measured the $\mathrm{C}-\mathrm{H}$ stretches in cyclohexane with Raman frequencies in the $2800-3000 \mathrm{~cm}^{-1}$ window. Figure 8 (a) shows the measured CARS signal as a function of the Stokes wavelength $\lambda_{s}$ and the relative delay $\Delta \tau$ of the pump pulse in which no chirp was applied. Because of the poor spectral resolution associated with femtosecond pump and Stokes pulses, it was not possible to resolve the two major Raman peaks separated by approximately $70 \mathrm{~cm}^{-1}$. Although the pulsewidth of the fundamental soliton does not change significantly when the wavelength is tuned by SSFS, chromatic dispersion in the fiber introduces pulse propagation delay as the function of the wavelength. This differential delay is compensated during the measurement by a variable delay stage in the pump path. In our experimental setup without pulse chirping the differential pulse 

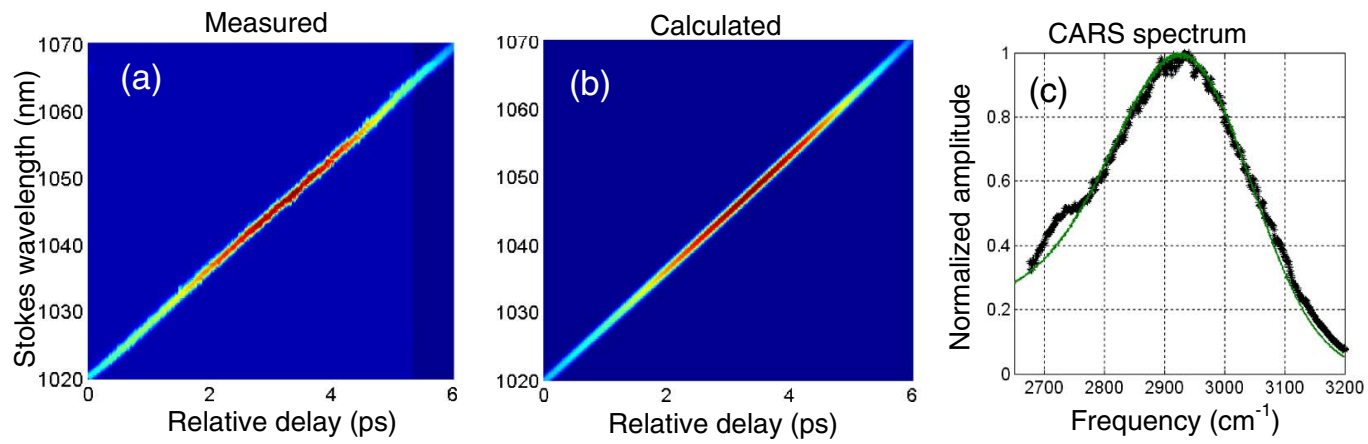

Fig. 8. (a) Measured and (b) calculated CARS as a function of pulse delay and the Stokes wavelength. (c) Measured (dots) and calculated (continuous line) CARS spectrum. No chirp was applied for pump and Stokes pulses.

delay is created primarily by the PCF. Because of the short pulses, temporal synchronization between pump and Stokes has to be nearly exact to produce any nonlinear mixing, so the measured CARS signal shown in Fig. 8(a) is a thin straight line with slope almost identical to the differential pulse delay. Figure 8(b) shows the result of numerical calculation using Eqs. (1) $-(5)$. The calculation takes into account the dispersion parameters of the optical fiber and all other optical components in the system. The CARS spectrum was extracted from the three-dimensional spectrograms as the amplitude along the differential delay curve with the slope of $0.121 \mathrm{ps} / \mathrm{nm}$ necessary to maintain exact pulse synchronization, as shown in Fig. 8(c).

In order to improve the spectral resolution, the pump and the Stokes pulses were both linearly stretched. Figure $\underline{9(a)}$ shows the measured $\lambda_{s}$ vs. $\Delta \tau$ diagram for SRG, which was obtained when the pump passed one time through a $5 \mathrm{~cm}$ SF6 glass rod while the Stokes passed through the other
$5 \mathrm{~cm}$ SF6 glass rod three times in a foldback configuration. The pump and the Stokes pulses were stretched to approximately 425 and $660 \mathrm{fs}$, respectively. Since the spectral widths of the pump and the Stokes were 4.43 and $4.83 \mathrm{THz}$, the slopes of the chirped pulses were 96 and $137 \mathrm{fs} / \mathrm{THz}$, respectively, for the pump and the Stokes. Although these two chirped pulses are not ideally parallel in the time-frequency diagram, the two major Raman $\mathrm{C}-\mathrm{H}$ stretch peaks can be identified on the $\lambda_{s}$ versus $\Delta \tau$ diagram. The instantaneous frequency difference between the chirped pump and Stokes pulses was a function of the time delay between the pulses and changes with the time delay at a rate of $\sim 116.5 \mathrm{fs} / \mathrm{THz}$ or, equivalently, $0.0317 \mathrm{ps} / \mathrm{nm}$, approximated from the average pulse chirping rate. The relative time delay between pump and Stokes pulses also depends on the differential material dispersive delays in the fiber, SF6 glass rods, and other optical elements, in addition to its dependence on the delay line position. Note that the slope of the measured SRG signal lines in the spectrogram
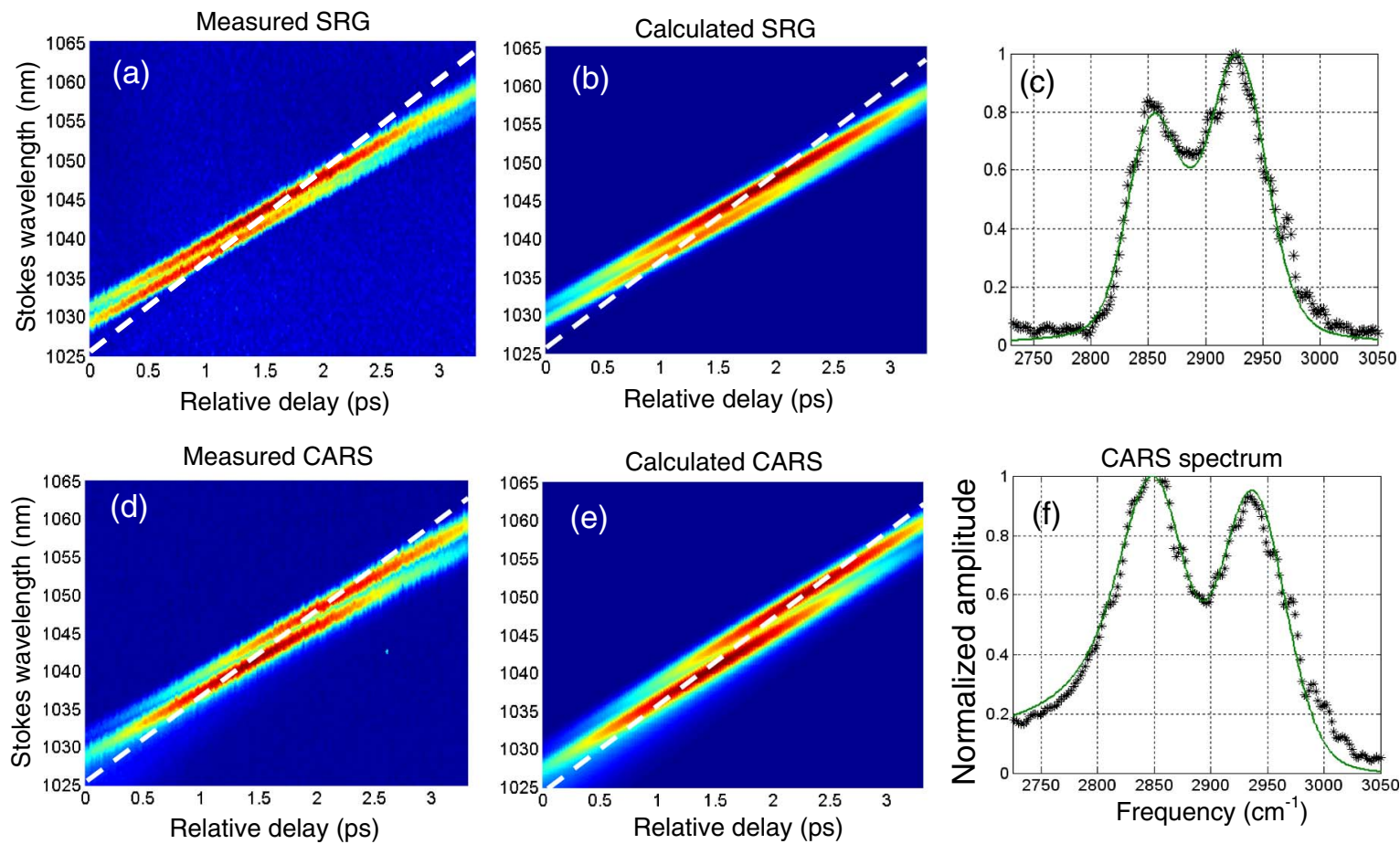

Fig. 9. (a) Measured and (b) calculated SRG signal as a function of pulse delay and the Stokes wavelength. (c) Measured (asterisks) and calculated (continuous line) SRG spectrum. (d) Measured and (e) calculated CARS signal as a function of pulse delay and the Stokes wavelength. (f) Measured (asterisks) and calculated (continuous line) CARS spectrum. Pump and Stokes pulses were chirped to 425 and 635 fs, respectively. Dashed lines in (a), (b), (d), and (e) indicate differential pulse delay. 
shown in Fig. 9(a) is approximately $0.1214 \mathrm{ps} / \mathrm{nm}$, which is

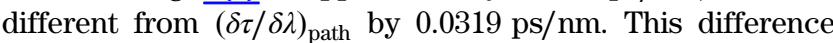
corresponds to the linear chirping applied to the pulses.

The dashed line in each spectrogram indicates the delay required in the pump path to exactly compensate for the differential delay of the Stokes pulses during a wavelength scan. It has the same slope $(\delta \tau / \delta \lambda)_{\text {path }}=0.0895 \mathrm{ps} / \mathrm{nm}$ as the bold line section indicated in Fig. 7(b). The SRG spectrum of cyclohexane was obtained by plotting the SRG signal measured along the dashed line with intercept chosen to maximize the signal amplitude. The result is shown in Fig. 9(c). It follows that a plot of SRG or CARS signal as a function of time delay at fixed Stokes wavelength would not give the correct spectrum. The calculated spectrum shown as a solid line in Fig. 9(c) agrees reasonably well with the measured results shown as asterisks in the same figure. Because of the low spectral resolution, the spectral peak at $2853 \mathrm{~cm}^{-1}$ is lower than that around $2939 \mathrm{~cm}^{-1}$, where two Raman lines are present.

For comparison, Figs. 9(d), 9(e), and 9(f) show the measured and calculated two-dimensional CARS spectrograms and the corresponding CARS spectrum. In the experiment, the CARS and the SRS were measured simultaneously by using the same setup, except that the CARS signal was obtained by integrating the total power of each anti-Stokes spectrum from the spectrometer. Since CARS measures the square of the absolute value of nonlinear susceptibility, it includes contributions from both the Raman resonance and the nonresonant background. Comparing Fig. $\underline{9(\mathrm{f})}$ with Fig. $\underline{9(\mathrm{c})}$, the resonance peak at $2853 \mathrm{~cm}^{-1}$ is higher in CARS than that in SRS. Apart from the impact of the nonresonant contribution, this is primarily caused by the spectral interference between the two major Raman resonances, which lowers the highfrequency peak for CARS. This interference also causes the minimum between the two Raman resonance peaks to be lower in CARS in comparison to SRG. The calculated CARS spectrum, in which nonresonant background was taken into account, matches reasonably well with the measured one as shown in Fig. 9(f), showing that the treatment described here adequately takes into account effects of spectral interferences and nonresonant background. This implies that the impact of nonresonant background can be extracted numerically.

To further improve the spectral resolution, we let the pump beam pass twice through a $6.5 \mathrm{~cm}$ thick SF6 glass, while the Stokes beam passed through a $5 \mathrm{~cm}$ thick glass five times, as illustrated in Fig. $\underline{6}$. In this case the chirped pump and the Stokes pulses were 855 and 1100 fs. Their slopes on the time-frequency diagram are 193 and $228 \mathrm{fs} / \mathrm{THz}$. (The average slope is approximately $0.057 \mathrm{ps} / \mathrm{nm}$.) The measured and calculated SRG and CARS spectra are shown in Fig. 10. In this measurement, the differential group delay of the Stokes pulse is $(\delta \tau / \delta \lambda)_{\text {path }}=0.058 \mathrm{ps} / \mathrm{nm}$ shown as the dashed line in the spectrogram, and the slope of the measured SRS and CARS signals is approximately $0.115 \mathrm{ps} / \mathrm{nm}$. With the increased pulse chirping compared to Fig. 9 , the two major Raman resonance peaks at 2853 and $2939 \mathrm{~cm}^{-1}$ can be clearly resolved in Fig. 10. The measured SRS and CARS spectra agree reasonably well with numerical calculations.

\section{DISCUSSION}

In our experiment, SRS and CARS spectra were measured simultaneously. This allows the comparison between Raman spectral shapes measured by these two techniques. Figure 11 shows a more systematic analysis of CARS and SRS spectra of cyclohexane (C-H stretches) simulated with different widths of stretched pulses for the pump and the Stokes.
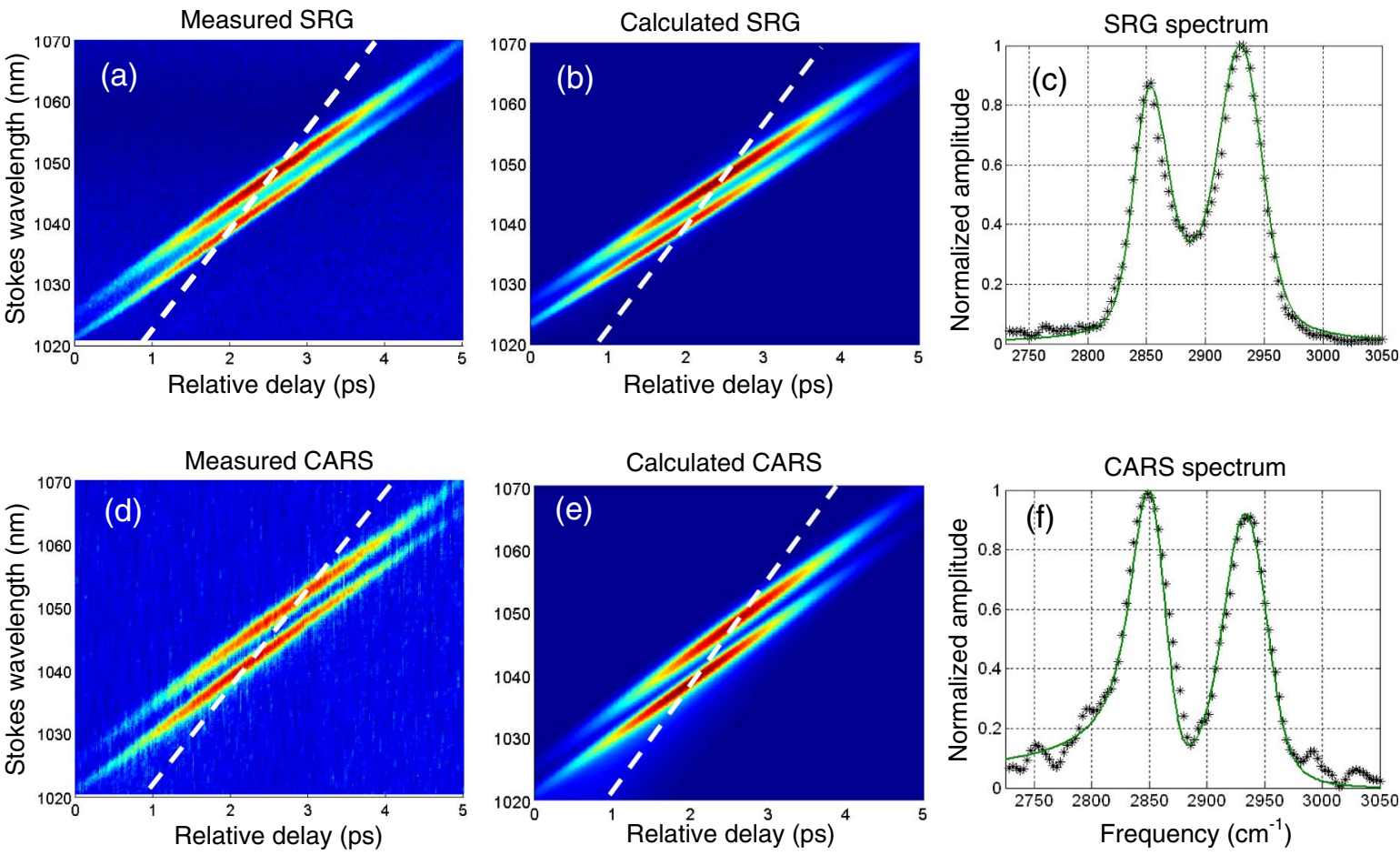

Fig. 10. (a) Measured and (b) calculated SRG as a function of pump pulse delay and the Stokes wavelength. (c) Measured (asterisks) and calculated (continuous line) SRG spectrum. (d) Measured and (e) calculated CARS signal as the function of pulse delay and the Stokes wavelength. (f) Measured (stars) and calculated (continuous line) CARS spectrum. Pump and Stokes pulses were chirped to 855 and 1055 fs, respectively. The dashed lines in (a), (b), (d), and (e) indicate differential pulse delay. 

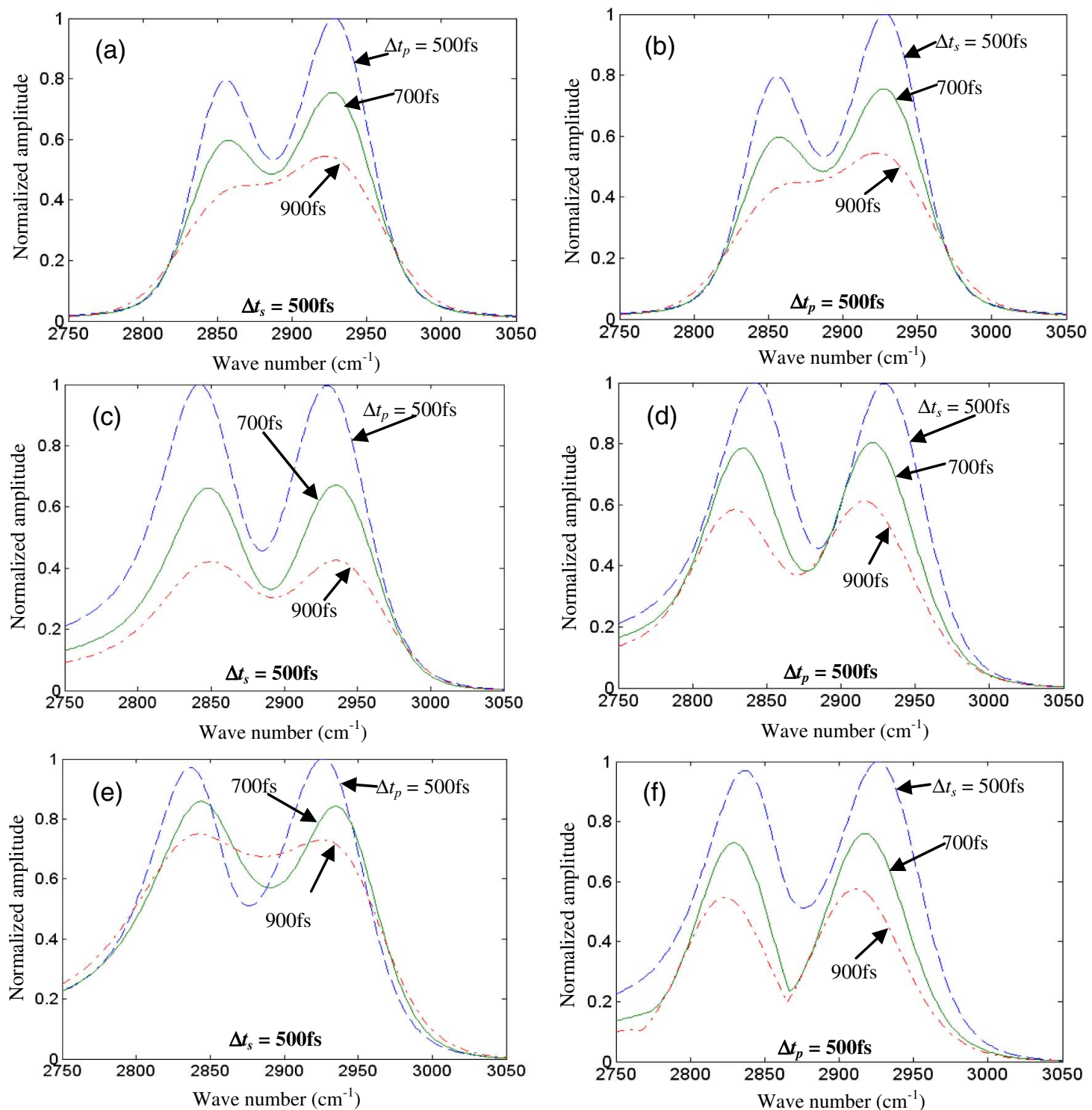

Fig. 11. Simulated spectra. (a) and (b) SRG. (c) and (d) CARS evaluated by the integrated anti-Stokes power. (e) and (f) CARS evaluated by the anti-Stokes peak spectral density. The horizontal axis is the pump-Stokes frequency separation. (a), (c) and (e) Fixed Stokes pulsewidth at $500 \mathrm{fs}$ but varying pump pulsewidths of 500, 700, and $900 \mathrm{fs}$. (b), (d), and (f) fixed pump pulsewidth at 500 fs but varying Stokes pulsewidths of 500, 700, and $900 \mathrm{fs}$.

The original widths of the transform-limited pump and Stokes pulses before chirping were both $100 \mathrm{fs}$, so that their spectral widths were identical. Assuming the pump and the Stokes pulses are perfectly synchronized in time with no relative delay, the frequency separation between the pump and the Stokes can be varied as the horizontal axis of Fig. 11. For the left column of Fig. 11 [Figs. 11(a), 11(c) and 11(e)] the Stokes pulsewidth was chirped to a fixed value of $500 \mathrm{fs}$ and the width of the pump pulse was varied from 500 to $900 \mathrm{fs}$; while for the right column of Fig. 11, [Figs. 11(b), 11(d) and 11(f)] the pump pulsewidth was chirped to a fixed value of $500 \mathrm{fs}$, and the width of the Stokes pulse was varied from 500 to 900 fs. Figures 11(a) and 11(b) show the calculated SRG as the function of pump-Stokes frequency separation. Comparison between Figs. 11(a) and 11(b) indicates that an increasing chirp on the pump or the Stokes pulses results in the same SRG spectral shape. However this may not be always true for the CARS. Figures $11(\mathrm{c})$ and $11(\mathrm{~d})$ show the
CARS spectra measured by the total anti-Stokes power (integrating the anti-Stokes power spectral density). Comparing Figs. 11(c) and 11(d), it is evident that the CARS spectral shapes can be different when the pump pulses are chirped longer than the Stokes pulses or when the Stokes pulses are chirped longer than the pump pulses. This is primarily attributed to insufficient spectral resolution and interference between the two Raman resonances in the CARS process. It is interesting to note that the CARS signal can also be measured by the maximum amplitude of the anti-Stokes spectral density as shown in Fig. 11(e) and 11(f), and the spectral shapes can be significantly different compared to the case where the total power of anti-Stokes was measured. Note that when the pump pulses were chirped more than Stokes pulses, the reduction in the total anti-Stokes power was more severe, as shown by comparing Figs. 11(c) and 11(d). However, the reduction in the maximum amplitude of the anti-Stokes spectral density was less severe when the pump pulses were chirped more 

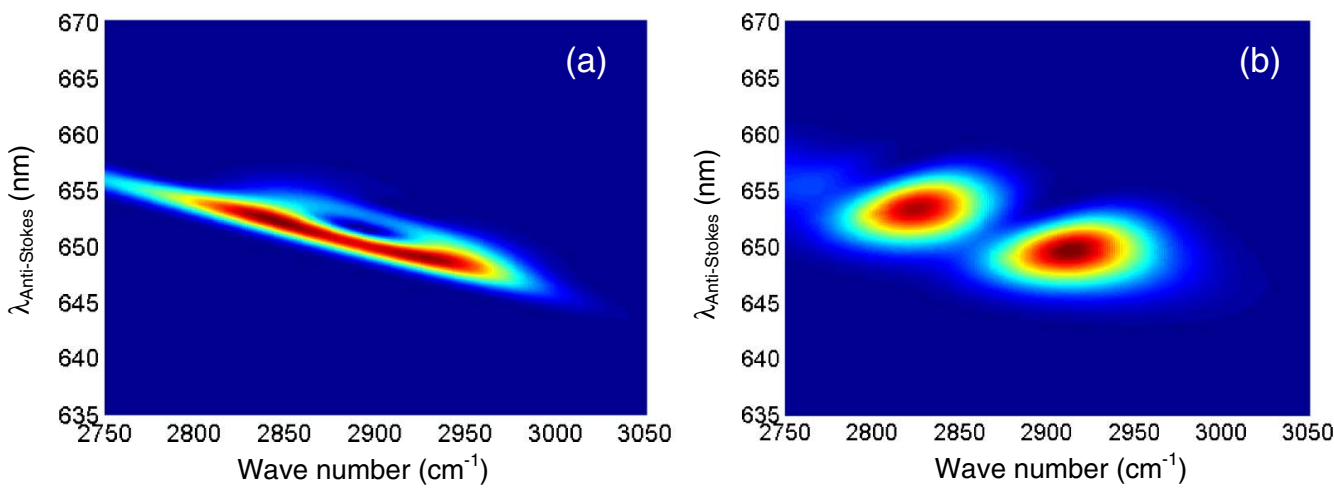

Fig. 12. Anti-Stokes spectral density calculated as a function of wavelength (vertical axis) and pump-Stokes frequency separation (horizontal axis), (a) with 500 fs Stokes pulsewidth and 900 fs pump pulsewidth, and (b) with 900 fs Stokes pulsewidth and 500 fs pump pulsewidth.

than Stokes pulses [compare Figs. 11(e) and 11(f)]. This was caused by spectral line narrowing of the anti-Stokes spectrum when the pump pulses were chirped more than the Stokes pulses. This is illustrated in Fig. 12, where the anti-Stokes spectral density was calculated for different pump-Stokes frequency separations. Other conditions are the same as those for Fig. 11. In Fig. 12(a) the Stokes and the pump pulsewidths were $\overline{500}$ and $900 \mathrm{fs}$, respectively. The spectral width of the anti-Stokes in Fig. 12(a) is obviously much narrower than that in Fig. 12(b) where Stokes pulsewidth was $900 \mathrm{fs}$ and the pump pulsewidth was $500 \mathrm{fs}$.

Due to the nonlinear nature of CRS, a tradeoff has to be made between the spectral resolution and the power efficiency, as the nonlinear mixing efficiency is reduced when the pulses are stretched. In previous CARS spectroscopy using spectral focusing, chirped broadband Stokes pulses were often used so that no wavelength tuning was required, and a frequency scan between the pump and the Stokes could be accomplished by a time scan $[16,17]$. However at any particular moment only a small fraction of the Stokes spectrum overlaps with the pump in the mixing process, while the Stokes power outside this time-overlapped region is wasted. In other cases $[8,15,18]$, including our excitation system, the temporal and spectral widths of the pump and the Stokes pulses are similar so that a maximum level of overlap between the pump and the Stokes pulses in the nonlinear mixing process is maintained, which maximizes the power efficiency. In addition, since the SRS amplitude is linearly proportional to the pump power while CARS is proportional to the square of the pump power, the detection sensitivity of SRS is less susceptible to pulse chirping in comparison to CARS as indicated by Fig. $\underline{4}$, which is an important consideration for applications with a low power budget.

\section{CONCLUSION}

In conclusion, we have systemically investigated CRS spectroscopy based on a single femtosecond fiber laser. SSFS in a nonlinear fiber allowed the Stokes wavelength to be continuously scanned through an electrical control. CARS and SRG spectra of cyclohexane were measured simultaneously at different levels of spectral focusing. As pulse optical power is a major limiting factor in this fiber-laser-based system, the tradeoff between spectral resolution and power efficiency is a major consideration. Our calculations indicate that pulse chirping is much more efficient than narrowband optical filtering for resolution improvement, and the power efficiency of SRS is less susceptible to pulse chirping in comparison to CARS. SRG and CARS spectra measured on $\mathrm{C}-\mathrm{H}$ stretches of cyclohexane were compared. Although the line shape of the CARS spectrum can be affected by interference between closely spaced Raman resonances and the nonresonant background, SRS is independent of these effects. The impact of differential pulse delay as a function of Stokes wavelength was carefully measured and presented in two-dimensional plots, and we show how to obtain the correct CARS or SRS spectrum as a cut through the two-dimensional plot. Measured results were well reproduced by numerical calculations, which ensure a good understanding of the physical mechanisms behind the experiments.

\section{ACKNOWLEDGMENTS}

We thank Peter Adany for the help in experimental system setup, and Paulette Spencer for use of the spontaneous Raman confocal microscope. This work was supported by the National Institutes of Health under grant NIH-RR023142.

\section{REFERENCES}

1. Y. R. Shen, The Principles of Nonlinear Optics (WileyInterscience, 2003).

2. M. D. Levenson and S. S. Kano, Introduction to Nonlinear Laser Spectroscopy (Academic, 1988).

3. J. X. Cheng, A. Volkmer, and X. S. Xie, "Theoretical and experimental characterization of coherent anti-Stokes Raman scattering microscopy," J. Opt. Soc. Am. B 19, 1363-1375 (2002).

4. C. W. Freudiger, W. Min, B. G. Saar, S. Lu, G. R. Holtom, C. He, J. C. Tsai, J. X. Kang, and X. S. Xie, "Label-free biomedical imaging with high sensitivity by stimulated Raman scattering microscopy," Science 322, 1857-1861 (2008).

5. J.-X. Chen and X. S. Xie, "Coherent anti-Stokes Raman scattering microscopy: instrumentation, theory, and application," J. Phys. Chem. B 108, 827-840 (2004).

6. F. Legare, C. L. Evans, F. Ganikhanov, and X. S. Xie, "Towards CARS endoscopy," Opt. Express 14, 4427-4432 (2006).

7. E. Ploetz, S. Laimgruber, S. Berner, W. Zinth, and P. Gilch, "Femtosecond stimulated Raman microscopy," Appl. Phys. B 87, 389-393 (2007).

8. E. R. Andresen, P. Berto, and H. Rigneault, "Stimulated Raman scattering microscopy by spectral focusing and fiber-generated soliton as Stokes pulse," Opt. Lett. 36, 2387-2389 (2011).

9. R. Hui and C. Johnson, "Laser system for photonic excitation investigation," U.S. patent 7,525,724 (April 28, 2009).

10. P. Adany, D. C. Arnett, C. K. Johnson, and R. Hui, "Tunable excitation source for coherent Raman spectroscopy based on a single fiber laser" Appl. Phys. Lett. 99, 181112 (2011). 
11. N. Nishizawa and T. Goto, "Widely wavelength-tunable ultrashort pulse generation using polarization maintaining optical fibers," IEEE J. Sel. Top. Quantum Electron. 7, 518-524 (2001).

12. J. R. Unruh, E. S. Price, R. Gagliano, L. Stehno-Bittel, C. K. Johnson, and R. Hui, "Two-photon microscopy with wavelength switchable fiber laser excitation," Opt. Express 14, 9825-9831 (2006).

13. J. P. Gordon, "Theory of the soliton self-frequency shift," Opt Lett. 11, 662-664 (1986)

14. P. Adany, E. S. Price, C. K. Johnson, R. Zhang, and R. Hui, "Switching of $800 \mathrm{~nm}$ femtosecond laser pulses using a compact PMN-PT modulator," Rev. Sci. Instrum. 80, 033107 (2009).

15. I. Rocha-Mendoza, W. Langbein, and P. Borri, "Coherent antiStokes Raman microspectroscopy using spectral focusing with glass dispersion," Appl. Phys. Lett. 93, 201103 (2008).

16. A. F. Pegorarol, A. Ridsdale, D. J. Moffatt, Y. Jia, J. P. Pezacki, and A. Stolow, "Optimally chirped multimodal CARS microscopy based on a single Ti:sapphire oscillator," Opt. Express 17, 2984-2996 (2009).

17. K. P. Knutsen, B. M. Messer, R. M. Onorato, and R. J. Saykally, "Chirped coherent anti-Stokes Raman scattering for high spectral resolution spectroscopy and chemically selective imaging," J. Phys. Chem. B 110, 5854-5864 (2006).

18. T. Hellerer, A. M. K. Enejder, and A. Zumbusch, "Spectral focusing: high spectral resolution spectroscopy with broadbandwidth laser pulses," Appl. Phys. Lett. 85, 25-27 (2004).

19. E. R. Andresen, C. K. Nielsen, J. Thøgersen, and S. R. Keiding, "Fiber laser-based light source for coherent anti-Stokes Raman scattering microspectroscopy," Opt. Express 15, 4848-4856 (2007).
20. A. F. Pegoraro, A. Ridsdale, D. J. Moffatt, J. P. Pezacki, B. K. Thomas, L. Fu, L. Dong, M. E. Fermann, and A. Stolow, "All-fiber CARS microscopy of live cells," Opt. Express 17, 20700-20706 (2009).

21. M. Baumgartl, T. Gottschall, J. Abreu-Afonso, A. Díez, T. Meyer, B. Dietzek, M. Rothhardt, J. Popp, J. Limpert, and A. Tünnermann, "Alignment-free, all-spliced fiber laser source for CARS microscopy based on four-wave-mixing," Opt. Express 20, 21010-21018 (2012).

22. M. Baumgartl, M. Chemnitz, C. Jauregui, T. Meyer, B. Dietzek, J. Popp, J. Limpert, and A. Tünnermann, "All-fiber laser source for CARS microscopy based on fiber optical parametric frequency conversion," Opt. Express 20, 4484-4493 (2012).

23. S. Lefrancois, D. Fu, G. R. Holtom, L. Kong, W. J. Wadsworth, P. Schneider, R. Herda, A. Zach, X. S. Xie, and F. W. Wise, "Fiber four-wave mixing source for coherent anti-Stokes Raman scattering microscopy," Opt. Lett. 37, 1652-1654 (2012).

24. Y. Ozeki, F. Dake, S. Kajiyama, K. Fukui, and K. Itoh, "Analysis and experimental assessment of the sensitivity of stimulated Raman scattering microscopy," Opt. Express 17, 3651-3658 (2009).

25. M. T. Cicerone, K. A. Aamer, Y. J. Lee, and E. Vartiainen, "Maximum entropy and time-domain Kramers-Kronig phase retrieval approaches are functionally equivalent for CARS microspectroscopy," J. Raman Spectrosc. 43, 637-643 (2012).

26. Y. Liu, Y. J. Lee, and M. T. Cicerone, "Broadband CARS spectral phase retrieval using a time-domain Kramers-Kronig transform," Opt. Lett. 34, 1363-1365 (2009).

27. K. Shi, P. Li, and Z. Liu, "Broadband coherent anti-Stokes Raman scattering spectroscopy in supercontinuum optical trap," Appl. Phys. Lett. 90, 141116 (2007). 